\title{
CP Violation Results from the NA48 experiments
}

\author{
Giuseppina Anzivino \\ Department of Physics, University of Perugia, via A. Pascoli, Perugia $(I){ }^{1}$
}

\begin{abstract}
.
The main goal of the NA48 experiments at the CERN SPS has been the search for direct CP violation (CPV) in kaon decays. In this article two selected results will be presented. In the neutral kaon sector, NA48 determined $\eta_{+-}$, related to the parameters of indirect and direct CPV ( $\eta_{+-}=$ $\varepsilon+\varepsilon^{\prime}$ ), from data collected during a dedicated run in 1999 with a pure $K_{L}$ beam. Using a sample of $47000 K_{L} \rightarrow \pi^{+} \pi^{-}$and 5 million $K_{L} \rightarrow \pi^{ \pm} e^{\mp} v$, we extract $\left|\eta_{+-}\right|=(2.223 \pm 0.012) \times 10^{-3}$. In the charged kaon decays, NA48/2 has measured the asymmetry $A_{g}=\left(g^{+}-g^{-}\right) /\left(g^{+}+g^{-}\right)$ of the linear slope parameter $g$ in the Dalitz plot of $K^{ \pm} \rightarrow 3 \pi$ decays from data collected in 2003 and 2004. Any non-zero value of $A_{g}$ would be evidence for direct CPV. A new technique of asymmetry measurement, involving simultaneous $K^{+}$and $K^{-}$beam and a large data sample allowed a result of unprecedented precision. The charge asymmetry parameter was measured to be $A_{g}^{c}=(-1.5 \pm 2.1) \times 10^{-4}$ with $3.11 \times 10^{9} K^{ \pm} \rightarrow \pi^{ \pm} \pi^{+} \pi^{-}$decays, and $A_{g}^{n}=(1.8 \pm 1.8) \times 10^{-4}$ with $9.13 \times 10^{7} K^{ \pm} \rightarrow \pi^{ \pm} \pi^{0} \pi^{0}$ decays. The results are compatible with the SM predictions, i.e. no evidence for direct $\mathrm{CP}$ violation at the order of $10^{-4}$ has been found.
\end{abstract}

Keywords: CP Violation, Kaon Decays

PACS: 13.20.Eb,13.25.Es

\section{EXPERIMENTAL SET-UP}

The data for $\eta_{+-}$measurement were taken during a dedicated run in 1999 with a pure $K_{L}$ beam produced by a $450 \mathrm{GeV}$ primary proton beam hitting a beryllium target. The neutral beam, after passing the final collimator, entered the decay volume which was followed by the NA48 detector. Two simultaneous focused kaon beams of opposite charge, with a momentum of $(60 \pm 3) \mathrm{GeV} / \mathrm{c}$ were used for the measurement of the charge asymmetry. The main components of the detector, described in detail in [1], are a Liquid Krypton Calorimeter ( $\mathrm{LKr}$ ) with tower read-out and a magnetic spectrometer. The calorimeter is an almost homogeneous ionization chamber with an active volume of $\sim 10 \mathrm{~m}^{3}$ and a $27 X_{0}$ thickness; it fully contains electromagnetic showers up to energies of $100 \mathrm{GeV}$ and has an energy resolution of $\sim 1 \%$ for photons of $20 \mathrm{GeV}$, and a spatial resolution of $\sim 1 \mathrm{~mm}$. The magnetic spectrometer, consisting of four drift chambers and a dipole magnet located between the second and the third chamber, has a spatial resolution of $100 \mu \mathrm{m}$.

\footnotetext{
${ }^{1}$ on behalf of the NA48 and NA48/2 Collaborations:Cagliari, Cambridge, CERN, Chicago, Dubna,
} Edinburgh, Ferrara, Firenze, Mainz, Northwestern, Perugia, Pisa, Saclay, Siegen, Torino, Warsaw, Wien. 


\section{CP VIOLATION PARAMETER $\eta_{+-}$}

The observable $\eta_{+-}$is related to the parameters of indirect and direct CPV ( $\eta_{+-}=$ $\left.\varepsilon+\varepsilon^{\prime}\right)$ and defined as the CP violating amplitude ratio of the neutral kaon decaying into two charged pions: $\eta_{+-}=A\left(K_{L} \rightarrow \pi^{+} \pi^{-}\right) / A\left(K_{S} \rightarrow \pi^{+} \pi^{-}\right)$. NA48 has determined $\left|\eta_{+-}\right|$via the measurement of the ratio of the decay rates

$$
R=\Gamma\left(K_{L} \rightarrow \pi^{+} \pi^{-}\right) / \Gamma\left(K_{L} \rightarrow \pi^{ \pm} e^{\mp} v\right)
$$

denoted as $\Gamma_{K 2 \pi} / \Gamma_{K e 3}$. The analysis is based on $47000 K_{L} \rightarrow \pi^{+} \pi^{-}$and five million $K_{L} \rightarrow \pi^{ \pm} e^{\mp} v$ decays. The selection is based on the requirement of good 2-track events for the two decays; then additional cuts are applied to suppress the dominant backgrounds to $K_{L} \rightarrow \pi^{+} \pi^{-}$, namely semileptonic $K_{e 3}$ and $K_{\mu 3}$ decays (Fig.1, left). For the selection of $K_{e 3}$ events, a cut on the $E / p$ is applied, where $E$ is the energy deposited in the $\mathrm{LKr}$ and $p$ is the track momentum measured in the magnetic spectrometer (Fig.1, right).
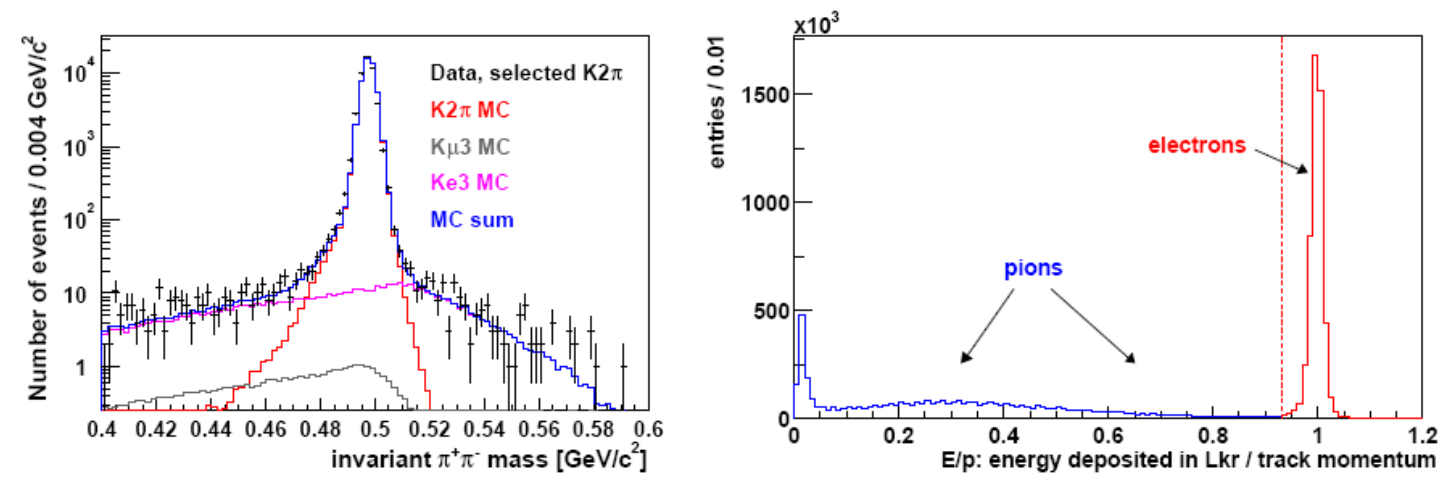

FIGURE 1. Left: Distribution of the invariant $\pi^{+} \pi^{-}$mass. Right: The E/p distribution for $K_{e 3}$ events.

The final result, after applying all the corrections is

$$
\frac{\Gamma\left(K_{L} \rightarrow \pi^{+} \pi^{-}\right)}{\Gamma\left(K_{L} \rightarrow \pi^{ \pm} e^{\mp} v\right)}=\left(4.835 \pm 0.022_{\text {stat }} \pm 0.016_{\text {syst }}\right) \times 10^{-3}=(4.835 \pm 0.027) \times 10^{-3} .
$$

Using the obtained ratio $\Gamma_{K 2 \pi} / \Gamma_{K e 3}$, we determined the $K_{L} \rightarrow \pi^{+} \pi^{-}$branching ratio, after subtraction of the contribution of the CP conserving direct emission process $K_{L} \rightarrow$ $\pi^{+} \pi^{-} \gamma(D E)(\sim 0.2)$ and including the $\mathrm{CP}$ violating inner bremsstrahlung component $K_{L} \rightarrow \pi^{+} \pi^{-} \gamma(I B)$ :

$$
B R\left(K_{L} \rightarrow \pi^{+} \pi^{-}\right)=(1.941 \pm 0.019) \times 10^{-3} .
$$

Finally we determine $\eta_{+-}$, using our result for the branching ratio of $K_{L} \rightarrow \pi^{+} \pi^{-}$ decay

$$
\left|\eta_{+-}\right|=\sqrt{\frac{B R\left(K_{L} \rightarrow \pi^{+} \pi^{-}\right)}{B R\left(K_{S} \rightarrow \pi^{+} \pi^{-}\right)} \cdot \frac{\tau_{K S}}{\tau_{K L}}}=(2.223 \pm 0.012) \times 10^{-3} .
$$

The results are in good agreement with recent measurements by KTeV [2] and KLOE [3] and all the three experiments contradict the 2004 PDG value [4]. 


\section{CP VIOLATION IN $K^{ \pm} \rightarrow 3 \pi$ DECAYS}

The $K^{ \pm} \rightarrow 3 \pi$ decays are usually described in terms of two Dalitz variables $u$ and $v: u=s_{3}-s_{0} / m_{\pi}^{2}$ and $v=s_{2}-s_{1} / m_{\pi}^{2}$, where $s_{i}=\left(p_{K}-p_{\pi i}\right)^{2}, p_{K}$ being the fourmomentum of the decaying kaon, and $s_{0}=\sum s_{i} / 3(i=1,2,3)$, index $i=3$ for the odd pion (the one with different charge from the other two). The matrix element can be expressed as a polynomial expansion of $u$ and $v$ :

$$
|\mathscr{M}(u, v)|^{2} \propto 1+g u+h u^{2}+k v^{2}
$$

where $g, h, k$ are the linear and quadratic Dalitz plot parameters $(|h|,|k| \ll|g|)$. Complementary with $\varepsilon^{\prime} / \varepsilon$, the $\mathrm{CP}$ observable in the charged kaon sector is the asymmetry

$$
A_{g}=\left(g^{+}-g^{-}\right) /\left(g^{+}+g^{-}\right) \approx \Delta g / 2 g
$$

of the linear slope parameter $g\left(g^{+}\right.$refers to $K^{+}, g^{-}$to $\left.K^{-}\right)$. Since there is no mixing in decays of charged kaons, any non-zero value of $A_{g}$ would reflect evidence for direct CPV. SM predictions for the charge asymmetry lay in the range $10^{-6}-10^{-5}$ [5], while theoretical calculations involving processes beyond the SM do not exclude substantial enhancements of $A_{g}$ [6]. The goal of NA48/2 was to measure $A_{g}$ with an accuracy of few $10^{-4}$.

The measurement of is based on the comparison of the $u$ distributions of $K^{+}$and $K^{-}$ decays. The density of $K^{ \pm}$events in the Dalitz plot is projected onto the $u$ axis to obtain one dimensional distributions $N^{ \pm}(u)$. Then the ratio

$$
R(u)=N^{+}(u) / N^{-}(u) \sim 1+\frac{\Delta g \cdot u}{1+g u+h u^{2}}
$$

is computed. Detector acceptance effects as well as variation in the response are canceled by design in NA48/2, being the two charged beams superimposed in space and present at the same time. This results in a large cancelation of systematic effects in the asymmetry measurement. However, the magnetic fields of the beam line (achromats, focusing quadrupoles, etc.) and of the spectrometer (dipole) are intrinsic source of charge asymmetry in the detector. In order to compensate for left-right asymmetries, the polarities of the relevant magnets along the beam line and the detector have been periodically reversed during the data taking. In two weeks an almost complete cycle, called super-sample, was completed. Each super-sample, treated as an independent unit in the analysis, contains four $K^{ \pm} \rightarrow 3 \pi$ samples with different combinations of beam line and spectrometer magnet polarities. The product of the four possible combinations forms a quadruple ratio

$$
R_{4}(u)=R(u)_{U S} \cdot R(u)_{U J} \cdot R(u)_{D S} \cdot R(u)_{D J}
$$

where the subscript $U(D)$ refers to a configuration in which $K^{+}$beam runs through the upper (lower) beam path in the achromatic magnet sets, while the subscript $S(J)$ refers to the spectrometer magnet polarity in which decay products, having the same charge as the corresponding beam, are deflected to the right (left) in the spectrometer (towards the Saleve (Jura) mountain). Finally, $\Delta g$ was extracted by fitting the quadruple ratio with a 

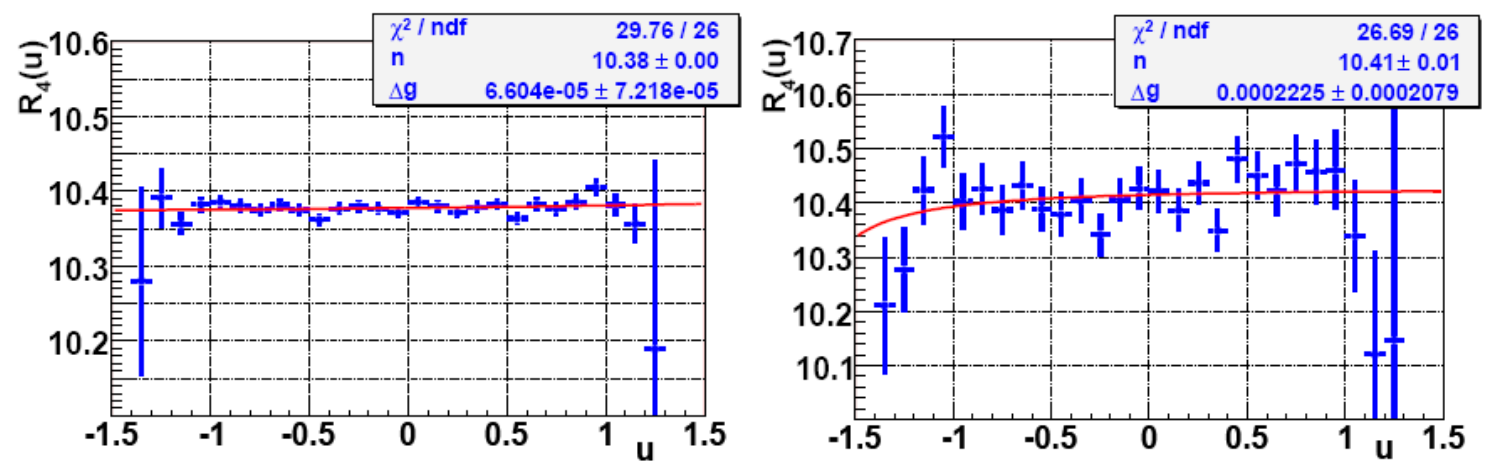

FIGURE 2. The quadruple ratio in bins of $u$ for charged (left) and neutral (right) mode, with the result of the fit to extract $\Delta g$.

function $f(u)=n \cdot\left(1+\frac{\Delta g u}{1+g u+h u^{2}}\right)^{4}$ and converted to the CP violating charge asymmetry, using the value of the Dalitz plot slope $g^{c}$, recently measured by NA48/2 [7], for the charged mode and the PDG value of $g^{n}$ for the neutral mode. In Fig.2 the final result for the fit is shown.

From the data samples taken in 2003 and 2004, 3.11 $\times 10^{9} K^{ \pm} \rightarrow \pi^{ \pm} \pi^{+} \pi^{-}$and $9.13 \times 10^{7} K^{ \pm} \rightarrow \pi^{ \pm} \pi^{0} \pi^{0}$ were selected. The results for the asymmetries are

$$
\begin{gathered}
A_{g}^{c}=\left(-1.5 \pm 1.5_{\text {stat }} \pm 0.9_{\text {trig }} \pm 1.1_{\text {syst }}\right) \times 10^{-4}=(-1.5 \pm 2.1) \times 10^{-4} \\
A_{g}^{n}=\left(1.8 \pm 1.7_{\text {stat }} \pm 0.9_{\text {syst }}\right) \times 10^{-4}=(1.8 \pm 1.8) \times 10^{-4} .
\end{gathered}
$$

The charge asymmetry parameter $A_{g}$ was determined, as proposed, with a total uncertainty of $\sim 2 \times 10^{-4}$ for each mode, which is an improvement in accuracy over the previous measurements [4] by more than an order of magnitude. NA48/2 precision is limited mainly by the available statistics. The results are compatible with the SM predictions, i.e. no evidence for direct $\mathrm{CP}$ violation at the order of $10^{-4}$ has been found. Due to the high precision achieved, the result can be used to constrain extensions of the $\mathrm{SM}$ predicting enhancements of the CP violating effects.

\section{REFERENCES}

1. V. Fanti et al., NA48 Collaboration, Nucl. Instrum. and Meth. A574, Elsevier, Amsterdam, 2007, pp. 433-471.

2. T. Alexopoulos et al., KTeV Collaboration, Phys. Rev. D70, APS, Maryland, 2004, pp. 092006-1092006-20.

3. F. Ambrosino et al., KLOE Collaboration, Phys. Lett. B638, Elsevier, Amsterdam, 2006, pp. 140-145.

4. S.Eidelman et al., Particle Data Group, Phys. Lett. B592, Elsevier, Amsterdam, 2004, 1.

5. E. Gámiz, J. Prades, I. Scimemi, JHEP 0310, 2003, pp 042-088;

G. Fäldt, E. P. Shabalin, Phys. Lett. B635, Elsevier, Amsterdam, 2006, pp. 295-298.

6. E. P. Shabalin ITEP preprint 8-98, 1998;

G. D’Ambrosio, G. Isidori, G. Martinelli Phys. Lett. B480, Elsevier, Amsterdam, 2000, pp. 64-170.

7. R. J. Batley et al., NA48/2 Collaboration, Phys. Lett. B649, Elsevier, Amsterdam, 2007, pp. 349-358. 